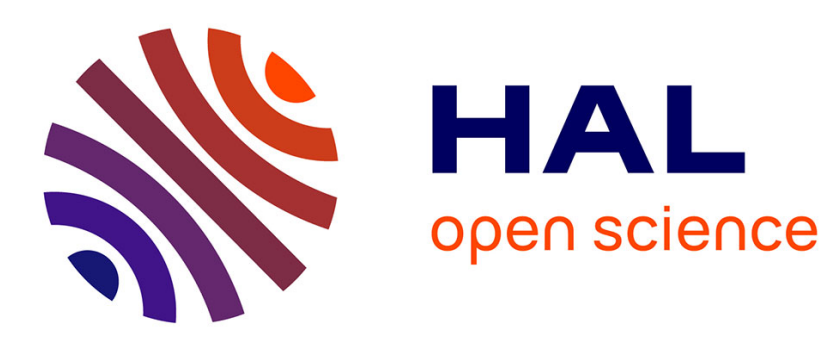

\title{
Acceleration Estimation Using Imperfect Incremental Encoders in Automotive Applications
}

Missie Aguado-Rojas, William Pasillas-Lépine, Antonio Loria, Alexandre de Bernardinis

\section{- To cite this version:}

Missie Aguado-Rojas, William Pasillas-Lépine, Antonio Loria, Alexandre de Bernardinis. Acceleration Estimation Using Imperfect Incremental Encoders in Automotive Applications. IEEE Transactions on Control Systems Technology, 2020, 28 (3), pp.1058-1065. 10.1109/TCST.2019.2896199 . hal02367536

\section{HAL Id: hal-02367536 https://hal.science/hal-02367536}

Submitted on 5 Mar 2020

HAL is a multi-disciplinary open access archive for the deposit and dissemination of scientific research documents, whether they are published or not. The documents may come from teaching and research institutions in France or abroad, or from public or private research centers.
L'archive ouverte pluridisciplinaire HAL, est destinée au dépôt et à la diffusion de documents scientifiques de niveau recherche, publiés ou non, émanant des établissements d'enseignement et de recherche français ou étrangers, des laboratoires publics ou privés. 


\title{
Acceleration estimation using imperfect incremental encoders in automotive applications
}

\author{
Missie Aguado-Rojas, William Pasillas-Lépine, Antonio Loría, and Alexandre De Bernardinis
}

\begin{abstract}
We address the problem of rotational velocity and acceleration estimation from incremental encoders in the presence of sensor imperfections, with a particular orientation towards automotive applications such as traction and brake control. In this area, measurements of the wheel speed and acceleration are often affected by large disturbances with a period of one revolution that arise from sensor imperfections and degrade the performance of most control designs. We present an algorithm to identify and remove such periodic perturbations online, without the need of error compensation look-up tables, and without assuming constant velocity. Experimental tests prove that the method is able to greatly reduce the impact of perturbations without introducing an important phase lag, as opposed to the results obtained using a notch-filter.
\end{abstract}

Index Terms - Velocity and acceleration estimation, incremental encoders, periodic measurement noise, timestamping algorithm, parameter estimation, antilock braking system.

\section{INTRODUCTION}

Real-time measurement of angular velocity and acceleration plays a crucial role in numerous control applications [1]. For example, in the area of traction and brake control, several vehicle-dynamics control systems rely on the measurement of the rotational speed of the wheels as the basic building block, making the wheel-speed sensor one of the most important sensors on a wheeled vehicle [2]. The most commonly used technology to measure rotational velocity and acceleration is based on incremental shaft encoders [3, $\S 6.2]$. They consist, mainly, in a toothed wheel (or a slotted disc) attached to the rotating shaft and a fixed pick-off sensor that detects the passing of the teeth and outputs a square wave signal in which each edge corresponds to the edge of one tooth. Thus, the velocity and acceleration are not directly measured, but they have to be reconstructed from the encoder pulses.

Several algorithms have been proposed to estimate velocity or acceleration from encoder measurements (see [4] and [5] for comprehensive reviews on the state-of-the-art), which can be classified either as model-based or as signal-based approaches.

The work of the first author is supported by CONACYT and SEP, Mexico.

M. Aguado-Rojas is with Université Paris-Sud, Universite ParisSaclay, L2S-CentraleSupélec, 91192 Gif-sur-Yvette, France (e-mail: missie.aguado@Is.centralesupelec.fr).

W. Pasillas-Lépine is with CNRS, L2S-CentraleSupélec, 91192 Gifsur-Yvette, France (e-mail: pasillas@l2s.centralesupelec.fr).

A. Loría is with CNRS, L2S-CentraleSupélec, 91192 Gif-sur-Yvette, France (e-mail: loria@I2s.centralesupelec.fr).

A. De Bernardinis is with SATIE, IFSTTAR-TEMA, 78000 Versailles, France (e-mail: alexandre.de-bernardinis@ifsttar.fr).
The former are well suited for applications for which simple and fairly accurate models exist, such as electrical motors. In the application of interest of this paper, however, the latter are usually preferred. In the field of automotive engineering accurate wheel models are rare because the dynamics is affected by the highly nonlinear and uncertain tyre-road friction characteristic. For this reason, signal-based approaches are considered to be better suited for automotive applications [6, appx. B]. Nevertheless, since they rely on the output of a real sensor subject to manufacturing and assembly tolerances, their accuracy is inevitably affected by the encoder imperfections.

An ideal incremental encoder is characterized by identical and equidistant teeth (or slits) distributed over the encoder's code-track. In real devices, however, well-known encoder imperfections (see, e.g., [3, $\S$ 6.7] as well as [7]-[9]), such as non-ideal teeth and eccentricity of the code-track, result in inexact readings of displacement and affect the quality of the velocity and acceleration estimations. Moreover, since the measurement error caused by sensor imperfections is periodic over one mechanical revolution, both estimates contain large periodic perturbations whose frequency is locked with the rotational frequency of the shaft. In an academic context, the inexact encoder readings induced by sensor imperfections can be compensated for using error look-up tables (see, e.g., [10]). However, these are suited solely for a particular encoder and their construction requires the availability of a high-resolution reference sensor. In an industrial context, error estimation and compensation approaches have been proposed, but they work only when the speed is constant (see, e.g., [1]) or does not vary significantly within one revolution (see, e.g., [11]).

The available literature shows that, on the one hand, the problem of rotational velocity and acceleration estimation from ideal incremental encoder measurements is welladdressed and, on the other hand, the most significant error sources in the encoder output signals caused by sensor imperfections are well-known. However, to the best of our knowledge, only a small number of works have addressed the issue of the effects of encoder imperfections on the velocity and acceleration estimation using real-time filters. The presence of periodic disturbances with a period of one mechanical revolution was noticed in [12] in the context of experimental identification of engine-to-slip dynamics in a sport motorbike. An offline zero-phase adaptive notch-filter-based scheme was used to remove the first harmonic component of the velocity of the wheels. The causes of the disturbances were later identified in [13] and the adaptive notch-filter was applied in real time. Similarly, the phenomenon was noticed in wheel acceleration 
measurements in [14], in the context of experimental validation of a five-phase ABS algorithm. A dynamic notch filter was proposed in [15] to eliminate the periodic disturbances on the wheel acceleration and the effect of this filter on the delay margin of the system's feedback loop was analysed. More recently, a method to remove the wheel-speed disturbances caused by sensor imperfections was proposed in [11] to estimate the pedaling cadence in an electric bicycle. Under the assumption that the true speed is well approximated by the mean revolution speed (which does not hold, e.g. during sudden braking scenarios), a constrained batch least squares formulation was used to recursively estimate and compensate for the geometric errors of the toothed wheel of the encoder.

In this paper, we are interested in the estimation of the wheel angular velocity and acceleration for automotive applications such as the antilock braking system (ABS). It should be noted, however, that the domain of application of our contribution is by no means restricted to the automotive field. The paper is organized as follows. In Section II we present the outline of a signal-based approach to reconstruct velocity and acceleration from encoder measurements, known as the time-stamping algorithm (TSA). In Section III, we describe the main error sources in incremental encoders, as well as their effect on the position, velocity and acceleration measured via the TSA. The contributions of our work are presented in Sections IV to VII. First, we introduce three models that capture the effects of sensor imperfections on the measured position, velocity, and acceleration signals. By combining these models with the features of the TSA, we extend the work presented in [16] and propose a method to identify and remove the periodical disturbances introduced by sensor imperfections. Rather than filtering out only the first harmonic component of the disturbances (i.e. the component whose frequency is equal to the speed of the wheel) as in the previous references, the aim of our approach is to identify a given number of harmonic components and to use that information to recover reliable estimates of the velocity and acceleration of the shaft. The method proposes a trade-off between the smoothness of the signals and the length of the delay, which could be exploited in order to satisfy delay margin constraints imposed by control laws. The effectiveness of the estimation algorithm is validated on an experimental setup via open-loop tests, and its performance is compared with respect to the one of a notch filter. A case-study from the ABS literature is used to illustrate the usefulness of the estimation algorithm in closed-loop control applications. Concluding remarks and perspectives on future work are given in Section VIII.

\section{TIME-STAMPING ALGORITHM}

In this section we present the so-called time-stamping algorithm (TSA) [17], which is used throughout this work to reconstruct angular velocity and acceleration from the output pulses of an incremental encoder. This algorithm is closely related to the Savitzky-Golay filter [18] (see also [19], [20]) and the least squares fit observer [21]. It consists in capturing, via a high-resolution clock, the time instants in which the edges of the encoder pulses are detected, and using the information of the last $\mathrm{n}$ edges to approximate the evolution of the angular position with a polynomial of order $\mathrm{m}$. Each edge detection is called an encoder event.

Let $t_{i}$ and $\theta_{i}$ denote the time instant and position corresponding to the $i$ th encoder event, and let $k$ be the index of the most recent event. The position $\theta$ at the current time $t$ is modelled as

$$
\theta(t)=p_{m} t^{m}+p_{m-1} t^{m-1}+\ldots+p_{0},
$$

where the coefficients $p_{0}, \ldots, p_{m}$ are unknown. A regression problem is formulated for the last $n$ events as

$$
\boldsymbol{A x}=\boldsymbol{b}
$$

with $\boldsymbol{A} \in \mathbb{R}^{n \times(m+1)}, \boldsymbol{x} \in \mathbb{R}^{m+1}$, and $\boldsymbol{b} \in \mathbb{R}^{n}$ given by

$$
\begin{aligned}
& \boldsymbol{A}=\left[\begin{array}{ccccc}
t_{k-n+1}^{m} & t_{k-n+1}^{m-1} & \cdots & t_{k-n+1} & 1 \\
\vdots & \vdots & \vdots & \vdots & \vdots \\
t_{k}^{m} & t_{k}^{m-1} & \cdots & t_{k} & 1
\end{array}\right], \\
& \boldsymbol{x}=\left[\begin{array}{lllll}
p_{m} & p_{m-1} & \cdots & p_{1} & p_{0}
\end{array}\right]^{\top} \text {, } \\
& \boldsymbol{b}=\left[\begin{array}{lll}
\theta_{k-n+1} & \cdots & \theta_{k}
\end{array}\right]^{\top} .
\end{aligned}
$$

Given $n>m \geq 2$, equation (2) is solved for $\boldsymbol{x}$, in the least-squares sense, as

$$
\boldsymbol{x}=\left(\boldsymbol{A}^{\top} \boldsymbol{A}\right)^{-1} \boldsymbol{A}^{\top} \boldsymbol{b} .
$$

Estimates of the angular velocity $\omega$ and acceleration $\alpha$ are then obtained via analytic differentiation of (1) with respect to time as

$$
\begin{aligned}
& \omega(t)=\sum_{i=1}^{m} i p_{i} t^{i-1}, \\
& \alpha(t)=\sum_{i=2}^{m}(i-1) i p_{i} t^{i-2} .
\end{aligned}
$$

In what follows, the order of the polynomial is set to $m=2$. Under this choice, the algorithm presented here is equivalent to the one described in [14, appx. 1], which has been shown to yield a good performance in automotive applications.

\section{INFLUENCE OF ENCODER IMPERFECTIONS}

An ideal incremental encoder is characterized by identical and equidistant slits distributed over the encoder's code-track and, in the case of quadrature encoders, by two symmetrical output channels 90 degrees out of phase. That is, an ideal encoder is characterized by equally-spaced edge transitions of the output pulses. In real devices, however, well-known manufacturing and assembly imperfections introduce variations in the location of the edge transitions that lead to differentialand integral-type errors in the output signals.

The most significant error sources are: 1) cycle error, i.e., stochastic variations of the edge transitions due to nonidentical and unequally-distributed slits, as well as limitations and irregularities of the encoder's signal generation and sensing hardware; 2) pulse-width error, generated when the duty cycle of the output pulses is not exactly symmetrical; 3 ) phase error, generated when the phase between the two channels is not exactly 90 degrees; and 4) eccentricity or tilt of the encoder's code-track due to concentricity and assembly tolerances. 

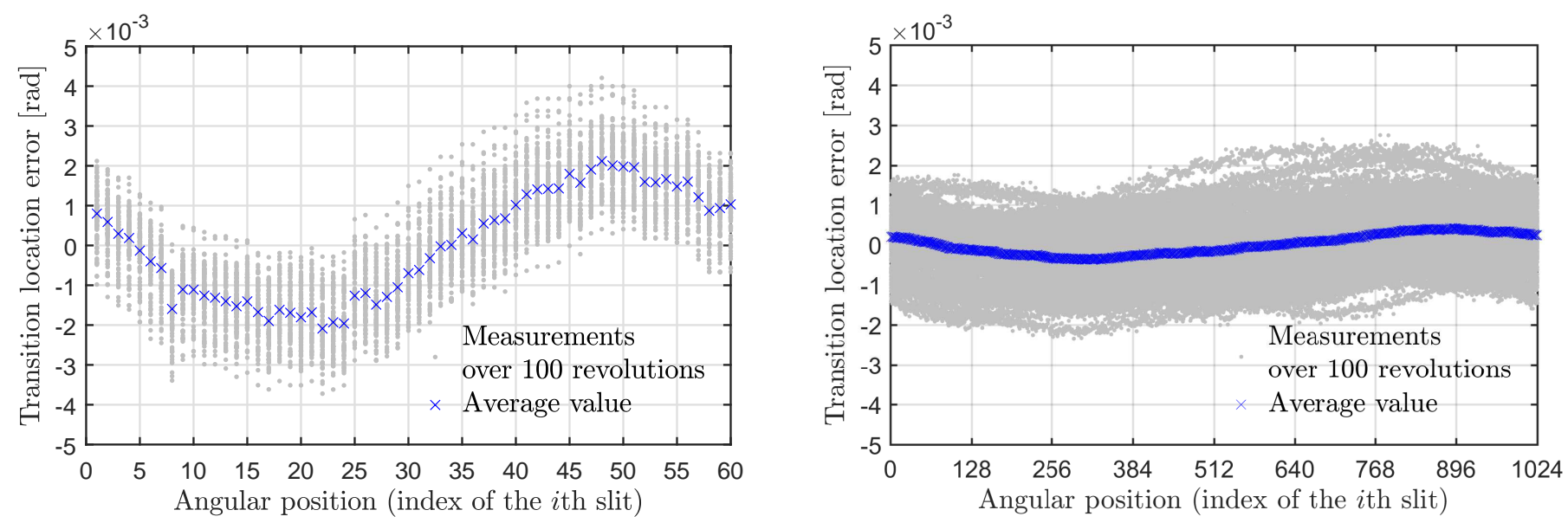

Fig. 1. Error in transition location as a function of angular position. LEFT: 60-ppr encoder. RIGHT: 1024-ppr encoder.

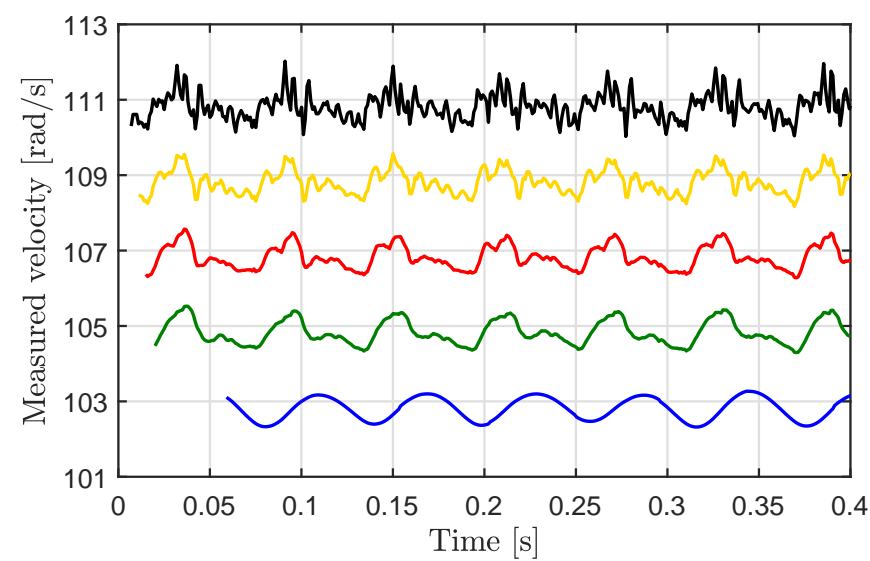

(a) Velocity: 6 events (black, offset $=4 \mathrm{rad} / \mathrm{s}$ ), 10 events (yellow, offset $=2 \mathrm{rad} / \mathrm{s}$ ), 15 events (red), 20 events (green, offset $=-2 \mathrm{rad} / \mathrm{s}$ ), 60 events (blue, offset $=-4 \mathrm{rad} / \mathrm{s}$ ).

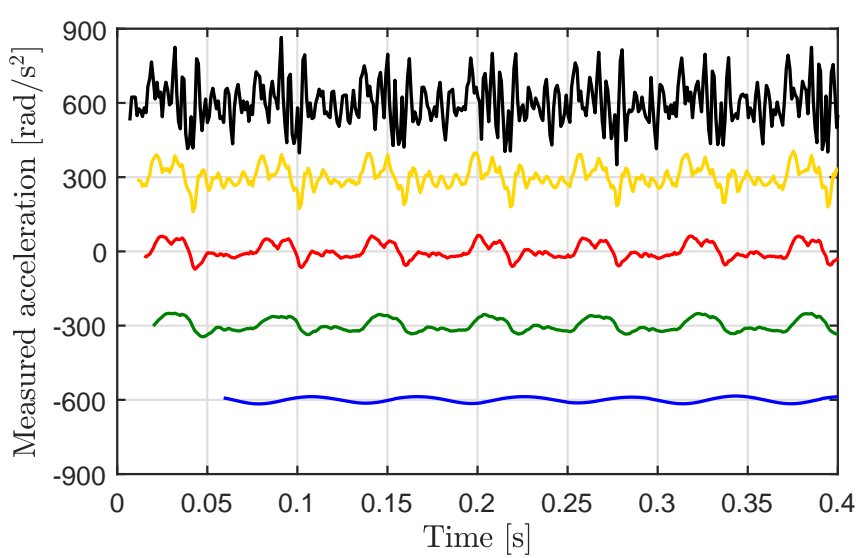

(b) Acceleration: 6 events (black, offset $=600 \mathrm{rad} / \mathrm{s}^{2}$ ), 10 events (yellow, offset $=300 \mathrm{rad} / \mathrm{s}^{2}$ ), 15 events (red), 20 events (green, offset $=-300$ $\mathrm{rad} / \mathrm{s}^{2}$ ), 60 events (blue, offset $=-600 \mathrm{rad} / \mathrm{s}^{2}$ ).

Fig. 2. Velocity and acceleration measured via the TSA for a $1020 \mathrm{rpm}(\approx 107 \mathrm{rad} / \mathrm{s})$ constant-velocity reference using a $60-\mathrm{ppr}$ encoder and different numbers of events (an offset is added to each signal for the sake of clarity).

The effects of these error sources have been thoroughly analyzed in [7]-[9], where it is shown that they can be modelled as two independent additive perturbations. That is, when viewed over the circumference of the encoder's codetrack, the first three error sources (also termed 'transition noise') appear as random high-frequency variations of the edge transition locations from their ideal values, whereas the fourth one introduces a systematic low-frequency variation with a period of one mechanical revolution - see also [13] and [16]. This is illustrated in Fig. 1, which shows the error in edgetransition locations for a 60-pulses-per-revolution (ppr) and a 1024-ppr encoder. These graphics were obtained using the experimental setup described in Section VI. The shaft of the encoders was rotated at a (theoretically) constant velocity and the time instants at which each edge transition was detected were stored and compared against the ideal time instants at which the edge transitions would have occurred if the encoders were perfect. The transition-location error was then computed via a least-squares fitting of the time-instant error and the theoretical value of the shaft velocity. For the $i$ th encoder slit, each dot represents the error measured at one of a total of 100 revolutions, and the cross represents the average value of the error corresponding to that slit. Both a once-per-revolution and a high-frequency stochastic variation of the transition locations can be observed.

The effects of the encoder imperfections on velocity and acceleration measurement are illustrated in Fig. 2. The graphic shows the measurements obtained via the TSA for an experiment with a constant reference of $1020 \mathrm{rpm}$ using different numbers of events. Note that, because the execution of the TSA is feasible only after the first $n$ events have been captured, the measured signals are not available at the beginning of the experiment. A clear periodicity can be observed in all cases. It can be noted as well that the amount of highfrequency variations that appear in the measured signals is directly related to the number $n$ of encoder events used in the estimation. Not surprisingly, because we are using a secondorder parabola to approximate the position history, the more angle/time points are used in the polynomial fit, the less the measured velocity and acceleration signals are prone to be affected by high-frequency transition location errors - see [20, Fig. 4]. This filtering effect introduces however an important 
phase lag when $n$ is too large, which hampers the ability of the TSA to capture small variations in the real signals and to accurately reflect large sudden variations.

\section{MeAsurement MOdELS}

The arguments given in the previous section and the experimental data shown in Fig. 1, naturally lead us to model the effects of sensor imperfections using a $2 \pi$-periodic function, that we denote $f_{r}$, added to the real position $\theta_{r}$. Hence the measured position $\theta_{m}$ corresponds to

$$
\theta_{m}=\theta_{r}+f_{r}\left(\theta_{r}\right) .
$$

If the function $f_{r}$ is approximated using a finite number $M_{r}$ of Fourier coefficients, one obtains

$$
\theta_{m}=\theta_{r}+\sum_{k=1}^{M_{r}}\left[a_{k}^{r} \sin \left(k \theta_{r}\right)+b_{k}^{r} \cos \left(k \theta_{r}\right)\right] .
$$

In what follows, we assume that both $a_{k}^{r} \ll 1$ and $b_{k}^{r} \ll 1$. Indeed, the error due to transition noise is often between 0.002 and 0.05 encoder cycles [22], whereas eccentricity of the codetrack is often between 0.05 and $0.25 \mathrm{~mm}$ [23]. Hence, this condition is always satisfied if the encoder is of reasonable quality and if the sensor has been correctly mounted on its shaft.

The fact that $\theta_{r}$ is unknown limits the applicability of the previous model [16]. Nevertheless, when the perturbation $f_{r}$ is sufficiently small, the function $\varphi_{r}$ that maps $\theta_{r}$ to $\theta_{m}$ through (5) is invertible. Denoting this inverse by $\varphi_{m}$, we have

$$
\theta_{m}=\theta_{r}+\left(f_{r} \circ \varphi_{m}\right)\left(\theta_{m}\right),
$$

where $\circ$ denotes the function composition operator. Defining $f_{m}=f_{r} \circ \varphi_{m}$, we obtain

$$
\theta_{m}=\theta_{r}+f_{m}\left(\theta_{m}\right)
$$

The function $f_{m}$ is also $2 \pi$-periodic. Note, however, that the Fourier coefficients of a finite approximation of $f_{m}$, given by

$$
\theta_{m}=\theta_{r}+\sum_{k=1}^{M}\left[a_{k} \sin \left(k \theta_{m}\right)+b_{k} \cos \left(k \theta_{m}\right)\right],
$$

are in general different from those of $f_{r}$, even when $M=M_{r}$. Actually, in general $M \neq M_{r}$ and it might be necessary to take $M>M_{r}$ to obtain a good approximation.

The new approximation (6) is the starting point for our estimation algorithm. The differentiation with respect to time of the expression above leads to the velocity and acceleration measurement models

$$
\omega_{m}=\omega_{r}+\omega_{m} \sum_{k=1}^{M}\left[k a_{k}^{\prime} \cos \left(k \theta_{m}\right)-k b_{k}^{\prime} \sin \left(k \theta_{m}\right)\right]
$$

and

$$
\begin{array}{r}
\alpha_{m}=\alpha_{r}+\alpha_{m} \sum_{k=1}^{M}\left[k a_{k}^{\prime \prime} \cos \left(k \theta_{m}\right)-k b_{k}^{\prime \prime} \sin \left(k \theta_{m}\right)\right] \\
-\omega_{m}^{2} \sum_{k=1}^{M}\left[k^{2} a_{k}^{\prime \prime} \sin \left(k \theta_{m}\right)+k^{2} b_{k}^{\prime \prime} \cos \left(k \theta_{m}\right)\right],
\end{array}
$$

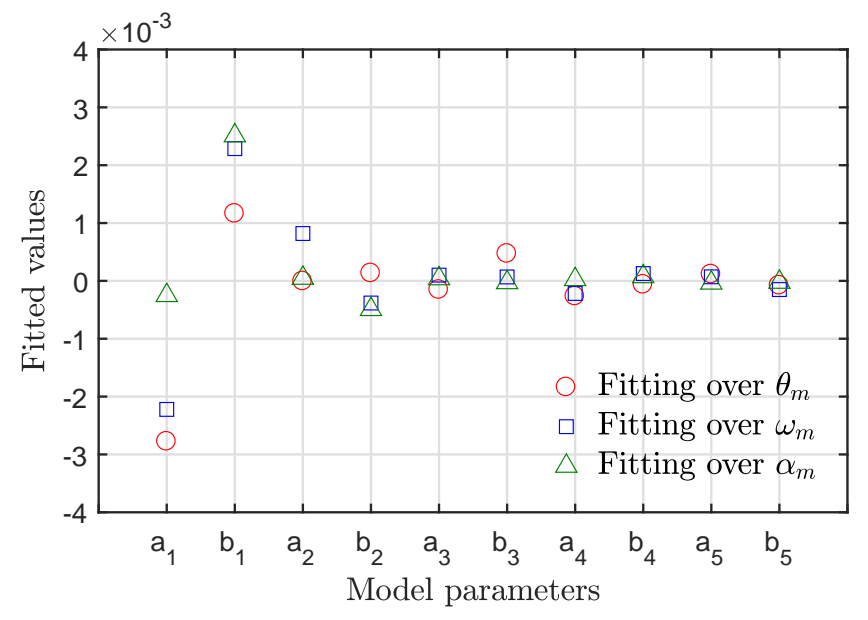

Fig. 3. Off-line least-squares fitting of the Fourier coefficients $a_{k}$ and $\boldsymbol{b}_{\boldsymbol{k}}$ for the measurement models (6), (7), and (8), using $\boldsymbol{n}=\mathbf{1 5}$ and $M=5$.

in which the Fourier coefficients $a_{k}^{\prime}, b_{k}^{\prime}, a_{k}^{\prime \prime}$ and $b_{k}^{\prime \prime}$ are unknown. Hence, our goal is to estimate the Fourier coefficients in order to recover reliable estimates of $\omega_{r}$ and $\alpha_{r}$.

Remark 1: The notation $(\cdot)^{\prime}$ and $(\cdot)^{\prime \prime}$ is used here in order to emphasize that the Fourier coefficients are different in (6), (7), and (8) because $\omega_{m}$ (resp. $\alpha_{m}$ ) does not correspond to the derivative of $\theta_{m}$ (resp. $\omega_{m}$ ), but it is obtained by using the TSA. This fact is illustrated in Fig. 3, where we show the values of $a_{k}$ and $b_{k}$ obtained via an offline least-squares fitting of the models (6), (7), and (8) with the data of the 1020 rpm experiment (Fig. 2) using 15 events. It can be observed that the fitted values of $a_{k}$ and $b_{k}$ vary when the fitting is performed using the different models, especially for the loworder harmonics.

In the experiments associated to Figs. 1 to 3 , an evaluation of the quality of these models as a function of the number of harmonics $M$ seems to indicate that the fit between data and the model improves until $M=\lceil N / n\rceil+1$, where $N$ is the number of pulses per revolution of the encoder, $n$ is the number of events used in the fit, and $\lceil\cdot\rceil$ is the smallest integer greater than or equal to the argument. Based on (7) and (8), an algorithm to reduce the periodic noise present in the measured signals can be designed as discussed in the following section.

\section{VELOCITY AND ACCELERATION ESTIMATION}

Let us rewrite (7) and (8) as

$$
\omega_{m}=\omega_{r}+\omega_{m} \boldsymbol{\phi}\left(\theta_{m}\right)^{\top} \boldsymbol{D} \boldsymbol{\vartheta}^{\prime}
$$

and

$$
\alpha_{m}=\alpha_{r}+\left[\alpha_{m} \phi\left(\theta_{m}\right)^{\top} \boldsymbol{D}-\omega_{m}^{2} \boldsymbol{\psi}\left(\theta_{m}\right)^{\top} \boldsymbol{D}^{2}\right] \boldsymbol{\vartheta}^{\prime \prime},
$$

where $\boldsymbol{D} \in \mathbb{R}^{2 M \times 2 M}, \phi$ and $\boldsymbol{\psi} \in \mathbb{R}^{2 M}$ are defined as

$$
\begin{aligned}
& \boldsymbol{D}=\operatorname{diag}[1,1,2,2, \ldots] \text {, } \\
& \phi\left(\theta_{m}\right)=\left[\begin{array}{lllll}
\cos \left(\theta_{m}\right) & -\sin \left(\theta_{m}\right) & \cos \left(2 \theta_{m}\right) & -\sin \left(2 \theta_{m}\right) & \cdots
\end{array}\right]^{\top}, \\
& \boldsymbol{\psi}\left(\theta_{m}\right)=\left[\begin{array}{lllll}
\sin \left(\theta_{m}\right) & \cos \left(\theta_{m}\right) & \sin \left(2 \theta_{m}\right) & \cos \left(2 \theta_{m}\right) & \cdots
\end{array}\right]^{\top},
\end{aligned}
$$




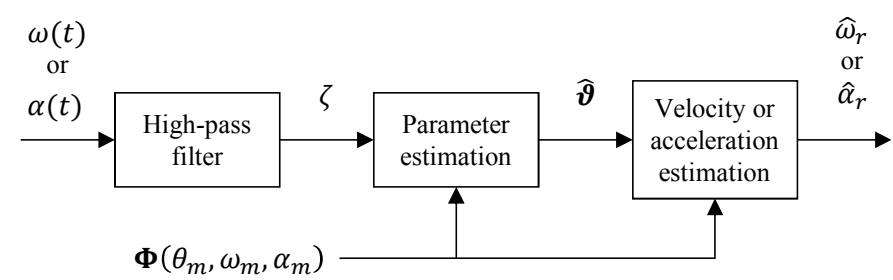

Fig. 4. Three-stage velocity and acceleration estimation scheme. The input signals $\omega(t)$ and $\alpha(t)$ are those obtained from the TSA - see (3) and (4).

and $\boldsymbol{\vartheta}^{\prime}, \boldsymbol{\vartheta}^{\prime \prime} \in \mathbb{R}^{2 M}$ contain the corresponding coefficients $a_{k}^{\prime}$, $b_{k}^{\prime}, a_{k}^{\prime \prime}$ and $b_{k}^{\prime \prime}$.

According to these models, provided that the frequencies of vehicle velocity and acceleration variations are much lower than those of the wheel harmonics, the measured velocity $\omega_{m}$ and the measured acceleration $\alpha_{m}$ can be seen as the sum of a low-frequency term $-\omega_{r}$ in (9) and $\alpha_{r}$ in (10)and a high-frequency (with respect to the first one) term $\boldsymbol{\Phi}\left(\theta_{m}, \omega_{m}, \alpha_{m}\right)^{\top} \boldsymbol{\vartheta}$, where $\boldsymbol{\Phi} \in \mathbb{R}^{2 M}$ depends on the known signals $\theta_{m}, \omega_{m}$, and $\alpha_{m}$, and is linear in the unknown parameters $\vartheta \in \mathbb{R}^{2 M}$. More precisely, we use

$\boldsymbol{\Phi}= \begin{cases}\boldsymbol{D} \phi\left(\theta_{m}\right) \omega_{m} & \text { for the model (9) } \\ \boldsymbol{D} \phi\left(\theta_{m}\right) \alpha_{m}-\boldsymbol{D}^{2} \boldsymbol{\psi}\left(\theta_{m}\right) \omega_{m}^{2} & \text { for the model (10), }\end{cases}$

and

$$
\boldsymbol{\vartheta}= \begin{cases}\boldsymbol{\vartheta}^{\prime} & \text { for the model (9) } \\ \boldsymbol{\vartheta}^{\prime \prime} & \text { for the model (10). }\end{cases}
$$

In order to estimate the real velocity (resp. acceleration), we perform the three-stage algorithm illustrated in Fig. 4, which is based on the work presented in [16]. In the first stage, in order to separate $\boldsymbol{\Phi}\left(\theta_{m}, \omega_{m}, \alpha_{m}\right)^{\top} \boldsymbol{\vartheta}$ from the other terms in (9) (resp. (10)), the measured signal is filtered using a first-order high-pass filter with a cutoff frequency that is considerably below that of the wheel revolution (for example $1 \mathrm{~Hz}$ ).

In the second stage, assuming that the filters are ideal and they completely eliminate the low-frequency term of the measured signals, the Fourier coefficients of the periodic perturbation in (9) (resp. (10)) are estimated using the parametric model

$$
\zeta=\boldsymbol{\Phi}\left(\theta_{m}, \omega_{m}, \alpha_{m}\right)^{\top} \boldsymbol{\vartheta}
$$

via the normalized recursive least-squares algorithm with forgetting factor [24, Ch. 4]:

$$
\begin{array}{ll}
\dot{\hat{\boldsymbol{\vartheta}}}=-\boldsymbol{\Gamma} \boldsymbol{\Phi} \varepsilon, & \widehat{\boldsymbol{\vartheta}}(0)=\widehat{\boldsymbol{\vartheta}}_{0} \\
\dot{\boldsymbol{\Gamma}}=\beta \boldsymbol{\Gamma}-\frac{\boldsymbol{\Gamma} \boldsymbol{\Phi} \boldsymbol{\Phi}^{\top} \boldsymbol{\Gamma}}{1+\kappa \boldsymbol{\Phi}^{\top} \boldsymbol{\Phi}}, & \boldsymbol{\Gamma}(0)=\boldsymbol{\Gamma}_{0}=\boldsymbol{\Gamma}_{0}^{\top}>0 \\
\varepsilon=\frac{\boldsymbol{\Phi}^{\top} \widehat{\boldsymbol{\vartheta}}-\zeta}{1+\kappa \boldsymbol{\Phi}^{\top} \boldsymbol{\Phi}}, &
\end{array}
$$

where $\widehat{\vartheta} \in \mathbb{R}^{2 M}, \boldsymbol{\Gamma} \in \mathbb{R}^{2 M \times 2 M}, \varepsilon \in \mathbb{R}$, and $\kappa$ and $\beta$ are positive constants.

Note that due to the form of the parametric model (11) any other standard algorithm may be used. Note as well that, due to the form of $\phi\left(\theta_{m}\right), \boldsymbol{\psi}\left(\theta_{m}\right)$, and $\boldsymbol{D}$, the realtime implementation of the RLS algorithm can be greatly

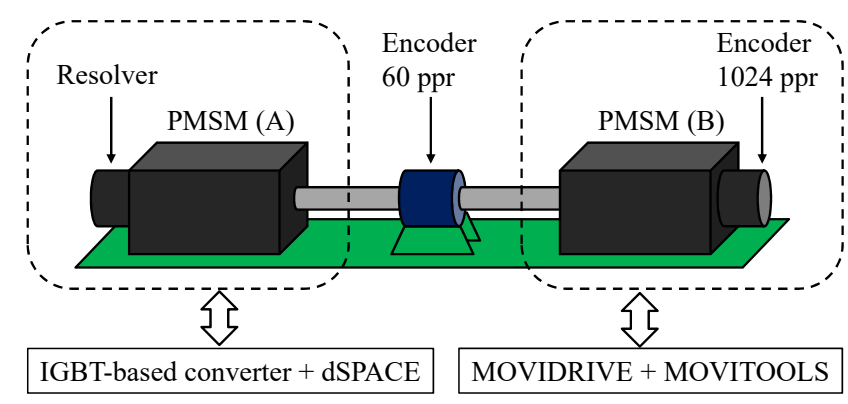

Fig. 5. Experimental setup. The low-resolution 60-ppr encoder and the high-resolution 1024-ppr encoder are those characterized in Fig. 1.

simplified, especially for large values of $M$. That is, the term $\boldsymbol{\Phi}^{\top} \boldsymbol{\Phi}$ in (13) and (14) simplifies to

$$
\boldsymbol{\Phi}^{\top} \boldsymbol{\Phi}= \begin{cases}\varsigma_{2} \omega_{m}^{2} & \text { for velocity estimation } \\ \varsigma_{2} \alpha_{m}^{2}+\varsigma_{4} \omega_{m}^{4} & \text { for acceleration estimation }\end{cases}
$$

where $\varsigma_{2}=\sum_{k=1}^{M} k^{2}$ and $\varsigma_{4}=\sum_{k=1}^{M} k^{4}$. Also, note that the regressor $\boldsymbol{\Phi}$ contains $M$ frequencies, that is, it is sufficiently rich to estimate the $2 M$ unknown parameters [24, Ch. 5].

Finally, using the estimated parameters $\widehat{\vartheta}$, we construct our velocity estimate as

$$
\widehat{\omega}_{r}=\omega_{m}-\omega_{m} \phi\left(\theta_{m}\right)^{\top} \boldsymbol{D} \widehat{\boldsymbol{\vartheta}}^{\prime}
$$

and our acceleration estimate as

$$
\widehat{\alpha}_{r}=\alpha_{m}-\left[\alpha_{m} \boldsymbol{\phi}\left(\theta_{m}\right)^{\top} \boldsymbol{D}-\omega_{m}^{2} \boldsymbol{\psi}\left(\theta_{m}\right)^{\top} \boldsymbol{D}^{2}\right] \widehat{\boldsymbol{\vartheta}^{\prime \prime}} .
$$

The experimental validation of this algorithm is presented in the following section.

\section{EXPERIMENTAL VALIDATION}

The experimental setup is illustrated in Fig. 5. It consists of two identical three-phase synchronous motors coupled via a torque transducer. The motor A has a built-in 2-pole resolver; it is driven by an IGBT-based converter and a dSPACE DS1006 processor board. The motor B has a built-in 1024-ppr encoder; it is driven by a MOVIDRIVE inverter and the MOVITOOLS MotionStudio software from SEW-EURODRIVE. For the tests presented in this paper, the motor B was used as an active torque generator and the motor $\mathrm{A}$ as a passive load. The estimation algorithm was implemented using an available 60ppr encoder mounted between the motors that has a resolution close to $48 \mathrm{ppr}$, which is frequent in automotive applications (see, e.g. [25, pp. 90-93]). The 1024-ppr encoder was used as a high-resolution reference sensor. For simplicity, the TSA was implemented considering only the rising edge of one output channel. The use of quadrature mode is nevertheless straightforward.

The performance of the estimation algorithm is illustrated in Fig. 6 for a time-varying velocity reference, and in Fig. 7 for a constant reference. The parameters and initial conditions of the estimation algorithm were set as $\kappa=1, \beta=0.1, \widehat{\boldsymbol{\vartheta}}_{0}=0$, and $\boldsymbol{\Gamma}_{0}$ as a matrix of the form $\boldsymbol{\Gamma}_{0}=\operatorname{diag}\left[\gamma_{1}, \gamma_{1}, \gamma_{2}, \gamma_{2}, \ldots\right]$ with $\gamma_{1}>\gamma_{2}>\ldots>\gamma_{M}>0$. In all cases a comparison with respect to the results obtained using a notch filter, as 

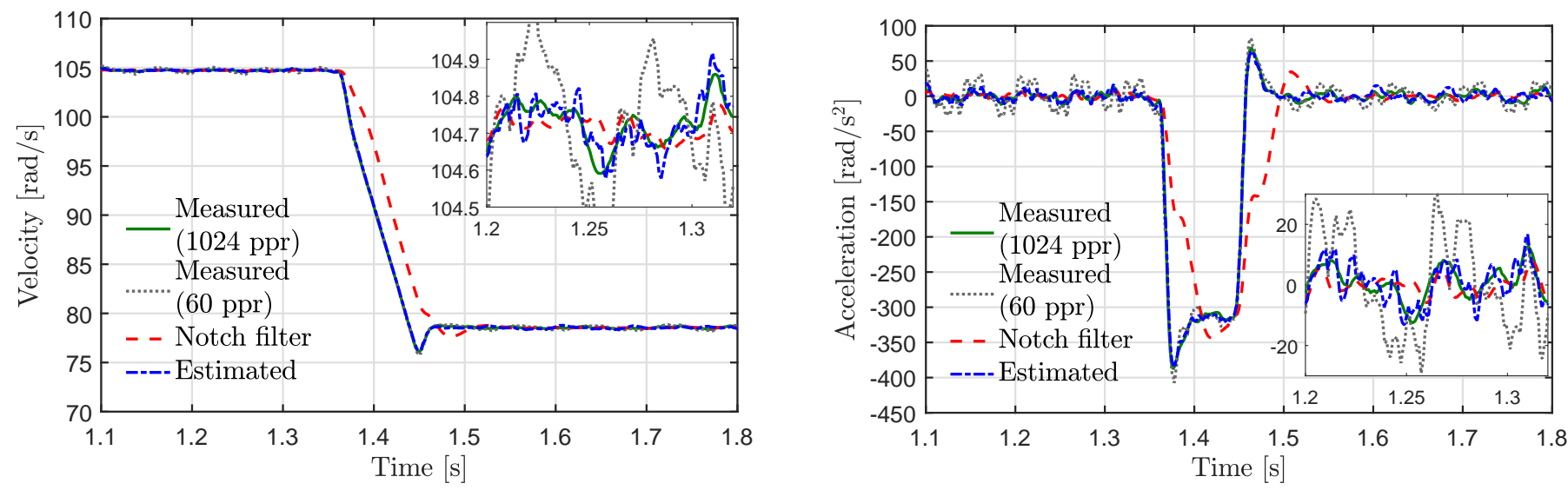

Fig. 6. Measured vs. filtered and estimated signals for a time-varying velocity reference using $n=\mathbf{1 5}$ and $M=\mathbf{5}$.
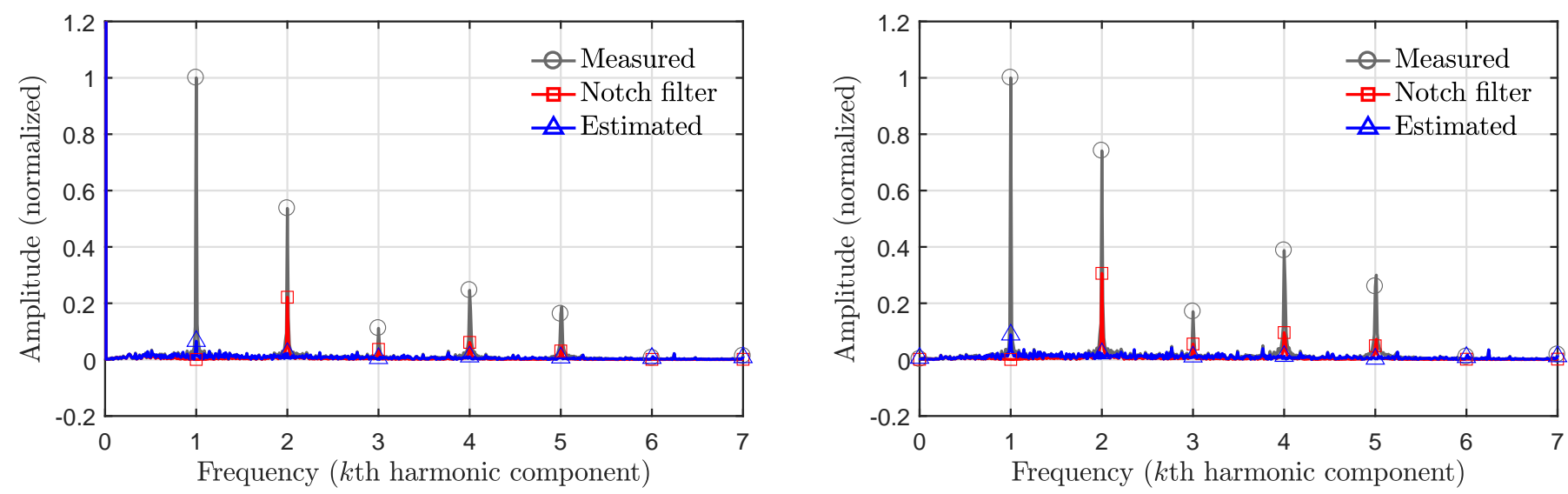

Fig. 7. Fourier transform of the measured, filtered, and estimated signals (LEFT: Velocity. RIGHT: Acceleration.) for a constant velocity reference using $n=15$ and $M=\mathbf{5}$. The depicted amplitude is normalized to the magnitude of the first harmonic of the measured signals.

proposed in [13] and [15], is shown. The damping ratio of the filter, $\xi \in(0,1)$, was chosen as $\xi=0.5$ in order to have a good compromise between the selectiveness of the filter and its response time. The signals labeled "measured" correspond to those obtained directly from the TSA.

The depicted results show that, once the estimated parameters have converged to appropriate values, the estimated signals contain significantly smaller oscillations than the measurements. This can be more easily noticed during the intervals in which the velocity or the acceleration are constant. During this intervals, the level of attenuation of the periodic perturbations of the estimation algorithm is similar to that of the notch filter. The usefulness of the estimation algorithm over a notch filter is, however, more evident during the intervals in which the velocity or the acceleration are changing. Even though both the notch filter and the estimation algorithm show a good performance in terms of reducing the amplitude of the oscillations, the filter clearly introduces a significant delay, whereas the estimation scheme follows the reference with no noticeable delay. The root-mean-square error (computed with respect to measurements of the 1024-ppr encoder) is given in Table I. In all cases, the proposed scheme performs better than both the TSA and the notch filter: it makes it possible to obtain better estimates of the velocity and of the acceleration without using a large number of events nor introducing a long delay. To further illustrate these advantages, in the next section we use the estimation algorithm in a concrete case-study of control systems technology, namely, the antilock braking system (ABS).

\section{CASE-STUdY: ABS CONTROL}

\section{A. System modelling}

Let us first present a simplified quarter-car model to describe the braking dynamics. For simplicity, only the forces acting on the longitudinal direction of a single loaded wheel are considered. Even though load transfer and combined slip are ignored, all the basic phenomena related to ABS appear in the model, and the limit cycles predicted by it are quite close to those obtained using more realistic models or experimental setups (cf. [14]).

Consider a wheel with radius $R$, inertia $I$, vertical load $F_{z}$, angular velocity $\omega$ and angular acceleration $\alpha$, mounted on a vehicle with longitudinal speed $v_{x}>0$ and longitudinal acceleration $a_{x}$. Define as state variables the wheel slip

$$
x_{1}=\left(R \omega-v_{x}(t)\right) / v_{x}(t),
$$

that represents the normalized relative velocity between the vehicle and the tyre, and the wheel acceleration offset

$$
x_{2}=R \alpha-a_{x}(t),
$$


TABLE I

ROOT-MEAN-SQUARE ERROR OF THE MEASURED, FILTERED, AND ESTIMATED SIGNALS RELATIVE TO THE 1024-PPR ENCODER.

\begin{tabular}{lccccc}
\hline \multirow{2}{*}{ Signal } & \multicolumn{2}{c}{ Time-varying reference } & & \multicolumn{2}{c}{ Constant reference } \\
\cline { 2 - 3 } \cline { 5 - 6 } & $\begin{array}{c}\text { Velocity } \\
{[\mathrm{rad} / \mathrm{s}]}\end{array}$ & $\begin{array}{c}\text { Acceleration } \\
{\left[\mathrm{rad} / \mathrm{s}^{2}\right]}\end{array}$ & & $\begin{array}{c}\text { Velocity } \\
{[\mathrm{rad} / \mathrm{s}]}\end{array}$ & $\begin{array}{c}\text { Acceleration } \\
{\left[\mathrm{rad} / \mathrm{s}^{2}\right]}\end{array}$ \\
\hline Measured (60ppr) & 0.1430 & 12.8528 & & 0.3007 & 30.0886 \\
Notch filter & 5.2597 & 33.5899 & & 3.0730 & 7.9528 \\
Estimated & 0.0783 & 5.9237 & & 0.0716 & 7.9405 \\
\hline
\end{tabular}

i.e. the relative acceleration between the vehicle and the tyre, and consider $v_{x}(t)$ and $a_{x}(t)$ as known external variables. During an ABS braking maneuver, the simplified dynamics of the system [26] is described by

$$
\begin{aligned}
& \dot{x}_{1}=\frac{1}{v_{x}(t)} x_{2} \\
& \dot{x}_{2}=-\frac{\bar{a}}{v_{x}(t)} \mu^{\prime}\left(x_{1}\right) x_{2}-\bar{b} u
\end{aligned}
$$

where $\bar{a}=\left(R^{2} / I\right) F_{z}, \bar{b}=(R / I) \gamma_{b}$, and $\gamma_{b}$ is an overall braking system gain. The control input $u=\dot{P}_{b}$ is the derivative of the brake pressure, and $\mu^{\prime}\left(x_{1}\right)$ denotes the derivative of the tyre-road friction coefficient $\mu$ with respect to $x_{1}$. The friction coefficient is modeled using Burckhardt's formula [27]

$$
\mu\left(x_{1}\right)=c_{1}\left(1-\exp \left(-c_{2} x_{1}\right)\right)-c_{3} x_{1},
$$

in which the parameters $c_{i}$ depend on the road conditions.

\section{B. Control strategy}

A five-phase hybrid ABS control algorithm, based on the one presented in [26], is described in Fig. 8. The objective of the algorithm is to keep $x_{1}$ in a small neighbourhood around its unknown optimal value $x_{1}^{\star}$, in order to minimize the braking distance while maintaining a good steerability of the vehicle. According to the active phase, the brake pressure is either kept constant (phases 2 and 5), or rapidly increased (phases 3 and 4) or decreased (phase 1). The changes in brake pressure are determined as a function of the wheel angular velocity $\omega$ and some parameters $u_{i}>0$ that depend on the maximum pressure variations allowed by the brake actuator. The switch from one phase to another is triggered when $x_{2}$ crosses some predefined thresholds $\epsilon_{i}>0$. Since $x_{2}$ depends on the value of the wheel acceleration $\alpha$, any perturbation in the measurement of $\alpha$ can cause the trigger to occur at inappropriate moments.

\section{Simulation results}

The performance of the control algorithm is illustrated in Figs. 9 to 11 , where the phase-plane behavior of the wheel dynamics during a braking maneuver of a vehicle running on dry concrete with an initial speed of $130 \mathrm{~km} / \mathrm{h}$ are shown. The TSA was implemented using $m=2, n=12$ and $M=6$, considering an encoder with parameters $N=60$ and $M_{r}=1$. The ideal limit cycle, obtained when the real values of $\omega$ and $\alpha$ are used in the control algorithm, is shown in all cases using green circles. The deceleration thresholds $\epsilon_{0}=50, \epsilon_{1}=30, \epsilon_{2}=40, \epsilon_{3}=20, \epsilon_{4}=20$, and

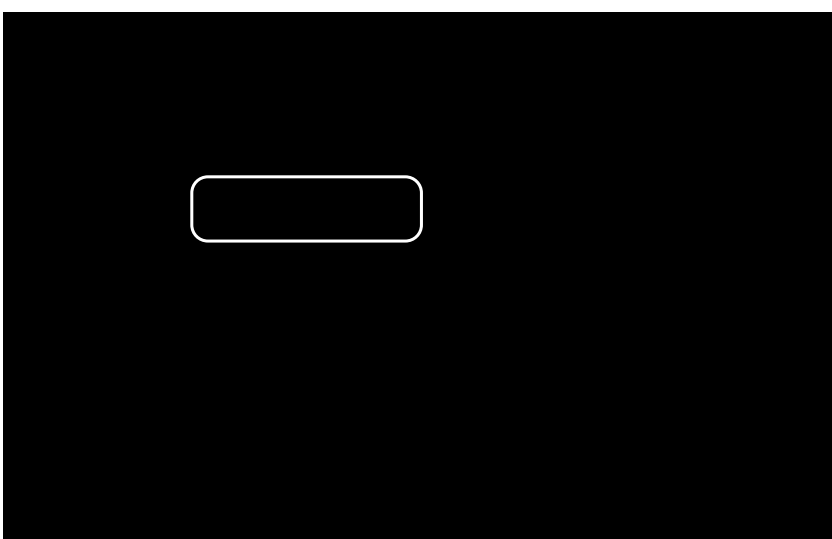

Fig. 8. Five-phase hybrid ABS control algorithm [26].

$\epsilon_{5}=30 \mathrm{~m} / \mathrm{s}^{2}$, are represented by the horizontal gray lines. The optimal point $\left(x_{1}^{\star}, 0\right)$ is indicated with a cross. Any variation in $x_{1}$ decreases the average braking force, while variations in $x_{2}$ demand a stronger effort from the brake actuator. Therefore, the amplitude of the cycle is a measure of the braking performance: the smaller the cycle, the shorter the vehicle's braking distance.

Fig. 9 shows the behavior obtained when the measurements affected by encoder imperfections are used. Due to the perturbations in the measured signals, the algorithm fails to cycle and remains blocked in an arbitrary phase (in this particular simulation, the wheel is trapped at the point $(-7,0))$. To cope with this, one may increase the values of $\epsilon_{i}$ (as suggested in [14]) so that only the most significant variations in $x_{2}$ are detected, but this implies increasing the amplitude of the limit cycle as well. When a notch filter (see [13] and [15]) is used to remove the perturbations in the measured signals (Fig. 10), the control algorithm is able to cycle around the desired point, but the performance is far from optimal. The delay introduced by the notch filter causes the algorithm to switch from one phase to another at inappropriate moments and the acceleration to go far outside the predefined thresholds. Finally, when the estimation algorithm is used to compensate for the encoder imperfections (Fig. 11), the control algorithm is able to cycle around the desired point. Although the estimation is not perfect and the acceleration violates the predefined thresholds, the excursion is smaller (around $35 \mathrm{~m} / \mathrm{s}^{2}$ ) than with the notch filter. Thus, the use of the estimation algorithm renders the performance of the control algorithm much closer to optimal.

\section{CONCLUSION}

This paper presented a three-stage estimation algorithm to estimate angular velocity and acceleration from incremental encoder measurements and reduce the effects of the encoder imperfections. The performance of the proposed algorithm has been evaluated in open-loop via experimental tests with satisfactory results, and in closed-loop via numerical simulations. Future work will focus on the real-time implementation of the estimation scheme in closed loop with ABS-related control and estimation algorithms, as well as on the potential utility of the method in different (non automotive) areas of application. 


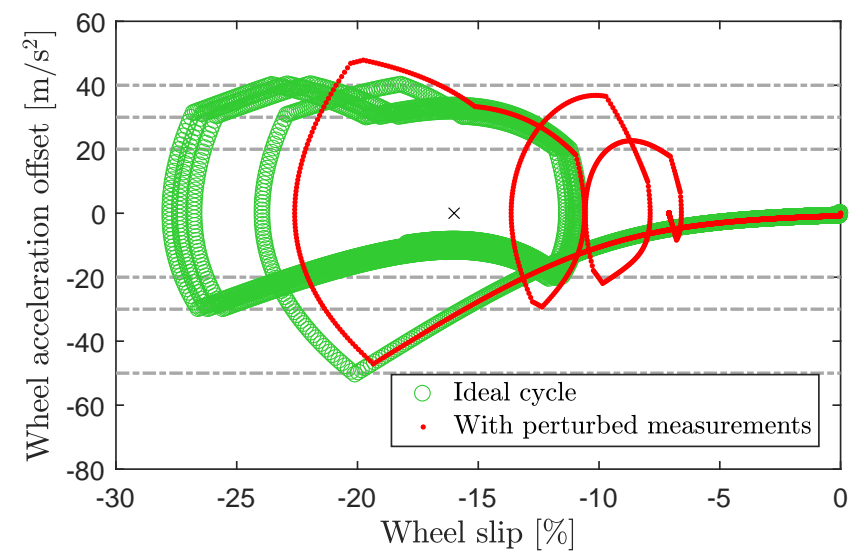

Fig. 9. Ideal cycle vs. behavior obtained with the measurements affected by encoder imperfections.

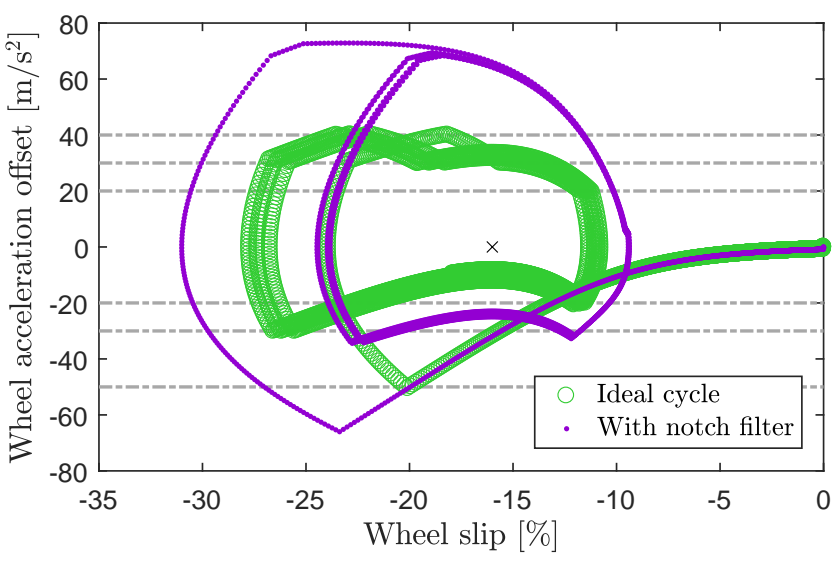

Fig. 10. Ideal cycle vs. behavior obtained using a notch filter.

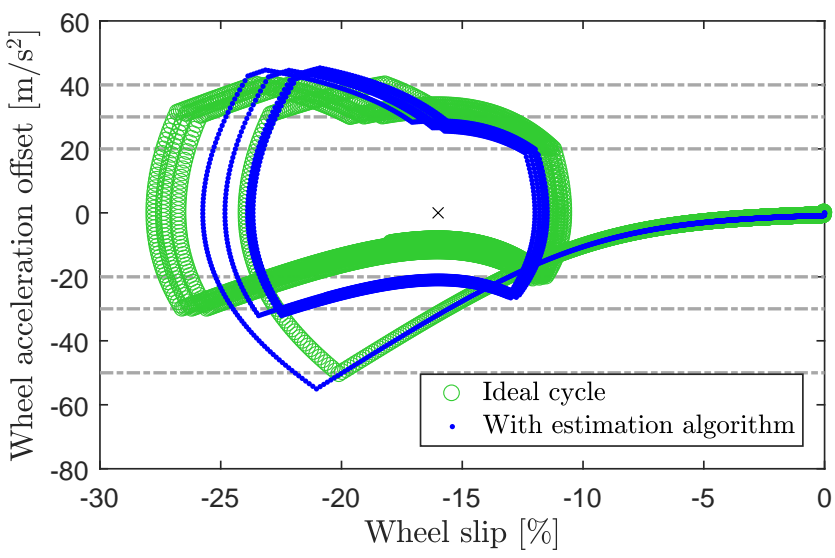

Fig. 11. Ideal cycle vs. behavior obtained using the estimation algorithm.

\section{REFERENCES}

[1] F. Gustafsson, "Rotational speed sensors: limitations, pre-processing and automotive applications," IEEE Instrumentation \& Measurement Magazine, vol. 13, no. 2, pp. 16-23, April 2010.

[2] — - "Automotive safety systems," IEEE Signal Processing Magazine, vol. 26, no. 4, pp. 32-47, July 2009.

[3] C. W. De Silva, Sensors and actuators: Engineering system instrumentation, 2nd ed. CRC Press, 2015.

[4] L. Bascetta, G. Magnani, and P. Rocco, "Velocity estimation: Assessing the performance of non-model-based techniques," IEEE Transactions on Control Systems Technology, vol. 17, no. 2, pp. 424-433, March 2009.
[5] R. Ronsse, S. M. M. De Rossi, N. Vitiello, T. Lenzi, M. C. Carrozza, and A. J. Ijspeert, "Real-time estimate of velocity and acceleration of quasi-periodic signals using adaptive oscillators," IEEE Transactions on Robotics, vol. 29, no. 3, pp. 783-791, June 2013.

[6] S. M. Savaresi and M. Tanelli, Active braking control systems design for vehicles, ser. Advances in Industrial Control. Springer-Verlag London, 2010.

[7] R. C. Kavanagh, "Shaft encoder characterization via theoretical model of differentiator with both differential and integral nonlinearities," IEEE Transactions on Instrumentation and Measurement, vol. 49, no. 4, pp. 795-801, Aug 2000.

[8] — - "Performance analysis and compensation of M/T-type digital tachometers," IEEE Transactions on Instrumentation and Measurement, vol. 50, no. 4, pp. 965-970, Aug 2001.

[9] _ - "Shaft encoder characterisation through analysis of the meansquared errors in nonideal quantised systems," IEE Proceedings Science, Measurement and Technology, vol. 149, no. 2, pp. 99-104, Mar 2002.

[10] K. K. Tan, H. X. Zhou, and T. H. Lee, "New interpolation method for quadrature encoder signals," IEEE Transactions on Instrumentation and Measurement, vol. 51, no. 5, pp. 1073-1079, Oct 2002.

[11] G. Rallo, S. Formentin, M. Corno, and S. M. Savaresi, "Real-time pedaling rate estimation via wheel speed filtering," IFAC-PapersOnLine, vol. 50, no. 1, pp. 6010-6015, July 9-14 2017, 20th IFAC World Congress.

[12] M. Corno and S. M. Savaresi, "Experimental identification of engineto-slip dynamics for traction control applications in a sport motorbike," European Journal of Control, vol. 16, no. 1, pp. 88 - 108, 2010.

[13] G. Panzani, M. Corno, and S. M. Savaresi, "On the periodic noise affecting wheel speed measurement," in Proceedings of the 16th IFAC Symposium on System Identification, vol. 45, no. 16, 2012, pp. 16951700.

[14] M. Gerard, W. Pasillas-Lépine, E. de Vries, and M. Verhaegen, "Improvements to a five-phase ABS algorithm for experimental validation," Vehicle System Dynamics, vol. 50, no. 10, pp. 1585-1611, 2012.

[15] T. B. Hoàng, W. Pasillas-Lépine, and A. De Bernardinis, "Reducing the impact of wheel-frequency oscillations in continuous and hybrid abs strategies," in Proceedings of the 11th International Symposium on Advanced Vehicle Control (AVEC'12), 2012.

[16] M. Aguado-Rojas, W. Pasillas-Lépine, A. Loría, and A. De Bernardinis, "Angular velocity estimation from incremental encoder measurements in the presence of sensor imperfections," IFAC-PapersOnLine, vol. 50, no. 1, pp. 5979-5984, July 2017, 20th IFAC World Congress.

[17] R. Merry, M. van de Molengraft, and M. Steinbuch, "Optimal higherorder encoder time-stamping," Mechatronics, vol. 23, no. 5, pp. 481-490, 2013.

[18] A. Savitzky and M. J. E. Golay, "Smoothing and differentiation of data by simplified least squares procedures," Analytical Chemistry, vol. 36, no. 8, pp. 1627-1639, 1964.

[19] R. W. Schafer, "What Is a Savitzky-Golay filter? [lecture notes]," IEEE Signal Processing Magazine, vol. 28, no. 4, pp. 111-117, July 2011.

[20] _ _ "On the frequency-domain properties of Savitzky-Golay filters," in 2011 Digital Signal Processing and Signal Processing Education Meeting (DSP/SPE), Jan 2011, pp. 54-59.

[21] R. H. Brown and S. C. Schneider, "Velocity observations from discrete position encoders," in Proc. SPIE 0858 Signal Acquisition and Processing, 1987, pp. 1111-1118.

[22] R. C. Kavanagh, "Improved digital tachometer with reduced sensitivity to sensor nonideality," IEEE Transactions on Industrial Electronics, vol. 47, no. 4, pp. 890-897, Aug 2000.

[23] D. Kreit, "Optical encoders - are they as accurate as you think?" Zettlex UK Ltd (Technical white paper), 2014.

[24] P. Ioannou and J. Sun, Robust Adaptive Control, ser. Dover Books on Electrical Engineering. Dover Publications, 2012.

[25] K. Reif, Ed., Brakes, brake control and driver assistance systems: Function, regulation and components, ser. Bosch Professional Automotive Information. Springer Vieweg, 2014.

[26] W. Pasillas-Lépine, "Hybrid modeling and limit cycle analysis for a class of five-phase anti-lock brake algorithms," Vehicle System Dynamics, vol. 44, no. 2, pp. 173-188, 2006.

[27] M. Burckhardt, Fahrwerktechnik: Radschlupf-Regelsysteme. VogelVerlag, 1993. 